\title{
Recomendações para a Gerência de Projetos no Desenvolvimento Distribuído de Software
}

\author{
Fábio Levy Siqueira, Paulo Sérgio Muniz Silva
}

\author{
Departamento de Engenharia de Computação e Sistemas Digitais - Escola Politécnica \\ da Universidade de São Paulo \\ Av. Prof. Luciano Gualberto, trav.3, $\mathrm{n}^{0} 158$ - 05508-900 - São Paulo - SP - Brasil \\ \{levy.siqueira, paulo.muniz\}@poli.usp.br
}

\begin{abstract}
Traditionally the software development is executed by physically nearby personnel. However, in some situations the stakeholders are separated through time and space by reasons that vary from the necessity to reduce costs to the necessity to use high qualified workers. Considering the difficulty involved in executing the management responsibilities in this kind of projects, a set of recommendations to the project management is proposed in this work. The present work shows the theoretical bases, employed method, and case study conducted to create and analyze these recommendations.
\end{abstract}

Resumo. O desenvolvimento de software é tradicionalmente realizado por pessoas localizadas em proximidade física. Entretanto em algumas situações as partes envolvidas estão separadas espacial e temporalmente devido a razões que variam desde a necessidade de reduzir custos até a necessidade de empregar uma mão-de-obra altamente qualificada. Abordando a dificuldade de realização das responsabilidades gerenciais em projetos desse tipo, neste trabalho são propostas recomendações para a gerência de projetos. Para isso são apresentados o embasamento teórico utilizado, o método empregado e o estudo de caso realizado para criá-las e analisá-las.

\section{Introdução}

O desenvolvimento de software não está limitado a uma equipe trabalhando em proximidade física dentro de um escritório. É possível unir pessoas em diversas localidades, sejam em diferentes bairros ou até em diferentes países, para realizar um desenvolvimento de software em conjunto. A esse desenvolvimento realizado por pessoas separadas espacial e temporalmente é dado o nome de desenvolvimento distribuído de software (DDS).

À primeira vista a realização de um DDS pode parecer algo relativamente simples. Os inúmeros avanços tecnológicos parecem diminuir as distâncias entre os trabalhadores. As novas formas de comunicação possibilitam o acesso a informações corporativas em alta velocidade, com pequena defasagem e uma quase ubiqüidade. As inovações existentes nas ferramentas de colaboração facilitam o trabalho em equipe, tornando possível uma interação virtual. Além dos avanços tecnológicos, existem também algumas estratégias e soluções técnicas e gerenciais que buscam diminuir os problemas e conflitos da dispersão espacial e temporal das pessoas [14] [19]. Mas, mesmo assim, o DDS continua a ser um desafio: da mesma forma que existem projetos 
de sucesso, também existem inúmeros relatos de projetos que fracassaram. A experiência prática mostra que a separação espacial e temporal das pessoas afeta profundamente o desenvolvimento de software, tornando mais difícil a sua realização devido a inúmeros motivos que variam desde a ausência de um idioma e uma faixa de horário comum a até a falta de confiança e de senso de equipe entre as pessoas envolvidas [6] [27]. Com isso não parece ser adequado tratar um projeto de software nesse tipo de situação como se fosse um projeto tradicional.

Um dos processos essenciais para qualquer projeto de desenvolvimento de software é a gestão de projetos. Cabe ao gerente de projetos equilibrar adequadamente 0 custo, o prazo, a qualidade e o escopo do projeto para satisfazer as expectativas e as necessidades das partes envolvidas. Para realizar isso, o gerente deve executar atividades como: desenvolver o cronograma, planejar os recursos, estimar o custo, desenvolver a equipe, identificar os riscos, entre diversas outras [29]. No entanto, em um desenvolvimento distribuído de software existem diversos obstáculos para o gerente realizar suas atividades, tais como: a dificuldade de planejar e criar um cronograma devido a separação temporal das pessoas, a necessidade de considerar custos de viagens e de investimento em infra-estrutura, a dificuldade de desenvolver a equipe devido a diferenças de culturas e idiomas, entre outros [3] [8]. Dessa forma, é necessária à gerência de projetos uma abordagem específica para o DDS para que as suas dificuldades e particularidades não levem o projeto ao fracasso.

Considerando as dificuldades do trabalho gerencial em um projeto de DDS, este trabalho propõe um método para criar um conjunto de recomendações básicas para a gerência de projetos. Estas recomendações buscam auxiliar a realização das atividades gerenciais ao considerar as mudanças necessárias devido às particularidades do DDS, levando em consideração os principais problemas apontados pelas experiências apresentadas em trabalhos existentes na literatura do assunto. Para mostrar a aplicação prática desse método, este trabalho também apresenta recomendações para os papéis de gerente de requisitos e de engenheiro de processos.

O artigo está estruturado da seguinte maneira: na seção 2 é discutido o desenvolvimento distribuído de software, apresentando algumas motivações e alguns problemas dessa forma de desenvolvimento. Na seção 3 é discutida a gerência de projetos no DDS, considerando a pesquisa atual sobre o assunto. Na seção 4 é apresentado o embasamento para a criação das recomendações, abordando a estrutura básica usada para definir as responsabilidades gerenciais e o método proposto para criar as recomendações. A partir disso, na seção 5 são apresentadas as recomendações para 0 gerente de requisitos e o engenheiro de processos. Para analisar as recomendações criadas, na seção 6 é apresentado o estudo de caso realizado. Por fim, na seção 7 é apresentada a conclusão, abordando algumas considerações sobre as recomendações.

\section{O Desenvolvimento Distribuído de Software}

Existem diversas motivações para realizar o desenvolvimento distribuído de software. Talvez a principal delas seja o acesso à mão-de-obra mais barata e ainda assim especializada disponível em países em desenvolvimento [6]. Mas, muitas vezes, é o oposto disso que motiva a realização de um DDS: a dificuldade em encontrar uma mãode-obra cara, que seja suficientemente qualificada ou que tenha o talento necessário para realizar determinadas atividades [18]. Além desses motivos, na literatura é possível 
encontrar diversas outras motivações como a diminuição de prazos ao aproveitar as diferenças de fusos horários para realizar um desenvolvimento em 24 horas [15], a possibilidade de se ficar mais próximo dos clientes [6], ou até a necessidade de dividir a equipe em diversas localidades devido ao tamanho do projeto [6].

Assim como existem diversas motivações para a realização de um DDS, existem diversos problemas relacionados a essa forma de desenvolvimento. Segundo Carmel [6], os principais problemas do DDS são: a dispersão geográfica, a perda da riqueza de comunicação, a quebra da coordenação, a falta de senso de equipe e as diferenças culturais. Mas além desses problemas é possível observar inúmeros outros em relatos de experiência existentes na literatura, tais como: a dificuldade de obtenção de vistos para viagens de negócio [21], a falta de horários comuns de trabalho [20], as diferenças de suporte técnico e de vendas de uma ferramenta em diferentes países [3], a falta de confiança entre as pessoas [4], a demora na resolução de problemas [20], entre outros.

Por mais que existam inúmeras motivações, vantagens e também problemas para a realização de um desenvolvimento distribuído de software, muitos dos atrativos e dificuldades estão diretamente ligados a algumas características do DDS. Dependendo do agrupamento, da distância física entre os grupos, da separação temporal entre as pessoas, das culturas regionais, dos idiomas, das diferenças dos locais, da infra-estrutura e da relação de negócio [31], o projeto terá algumas dificuldades e vantagens que podem ser irrelevantes em outros projetos. Essas particularidades farão com que os processos de desenvolvimento, os riscos e a estratégia da gerência sejam diferentes.

\section{A Gerência de Projetos e o Desenvolvimento Distribuído de Software}

Em projetos em que não se está realizando o desenvolvimento distribuído de software, as atividades gerenciais já são bastante complexas. Entre suas diversas responsabilidades, cabe ao gerente de projetos planejar, controlar e monitorar o andamento do que foi planejado e também motivar a equipe de desenvolvimento. Em um DDS, além dos problemas e dificuldades comuns a projetos em que as pessoas estão em proximidade física, existem inúmeros outros obstáculos para a realização das responsabilidades gerenciais, originários das características dessa forma de desenvolvimento. Como exemplo desses problemas e dificuldades, podem ser citados: a dificuldade de supervisionar e controlar o trabalho, pelo menos das formas tradicionais [12], a dificuldade de gerenciar conflitos [8] e a dificuldade de comunicação entre as partes devido a diferenças de idioma [27].

Ao considerar as várias dificuldades para a realização das atividades gerenciais, existem na literatura trabalhos que buscam auxiliar o entendimento dos desafios da gerência de projetos no DDS e, também, propor algumas soluções. Alguns desses trabalhos discutem as dificuldades e os problemas a partir da experiência obtida em um estudo de caso ou em um conjunto deles, como em [3] [6] [8] [20] [21]. Outros trabalhos tratam o assunto em um outro nível de abstração ao definir as linhas gerais das atividades do gerente, como é feito em [26] [33]. Ao invés de propor modelos para a atividade gerencial, outros trabalhos propõem a criação de ferramentas que buscam facilitar a execução de parte das responsabilidades desse papel, como em [11] [13].

Por mais que as ferramentas e os modelos propostos auxiliem a realização da atividade da gerência de projetos em um DDS, seus escopos muitas vezes cobrem 
apenas parte das responsabilidades gerenciais (coordenação e controle, principalmente). Mas, mais que isso, em muitos casos ou não são definidos as necessidades que originaram as propostas, ou os problemas levantados não parecem considerar muitas das questões apontadas por relatos de experiência. Com isso, parece ser essencial entender os diversos problemas existentes no DDS e suas possíveis soluções, criando um conjunto de requisitos que aproveitem o conhecimento existente na literatura.

\section{A Criação das Recomendações}

Considerando a pesquisa atual, neste trabalho se propõe a criação de um conjunto de recomendações básicas para a gerência de projetos. Com a definição de um conjunto de recomendações pretende-se permitir, no futuro, a elaboração de um modelo da gerência de projetos no DDS e uma proposta para uma ferramenta de apoio, levando em consideração os problemas e as soluções apontadas pela literatura e aquelas observadas empiricamente. Mas, para que quaisquer desses objetivos sejam razoáveis, é necessário haver uma quantidade suficiente de recomendações e pontos a serem modificados na gerência de projetos devido ao DDS. Com isso, parece ser importante realizar uma análise da influência dessa forma de desenvolvimento na gerência de projetos para que seja possível criar soluções mais abrangentes.

Além de ser uma base para outras propostas, um conjunto de recomendações pode ser facilmente empregado durante um projeto dada a sua natureza prática. Um gerente, ao observar quais recomendações são importantes ao projeto, pode segui-las conforme seja mais interessante e adequado como, por exemplo, ao alterar uma atividade, técnica ou método que seriam normalmente utilizados.

\subsection{As Responsabilidades da Gerência de Projetos}

O potencial de aplicação das recomendações nas mais diversas situações faz necessário utilizar como estrutura básica das atribuições do gerente de projetos uma referência com um nível de abstração suficiente para que não seja específico a uma determinada metodologia. Considerando essa necessidade, foi utilizado como guia das responsabilidades a vista da gerência de projetos baseada na NBR ISO/IEC 12207 [1]. Por ser uma norma com um nível alto de abstração, não são especificados detalhes de como executar as atividades definidas, havendo a liberdade para cada organização implantar o padrão da forma que seja mais adequada às suas necessidades.

O processo gerencial definido pela NBR ISO/IEC 12207 deve ser empregado especificamente para cada um dos processos fundamentais e de apoio, caso seja aplicável. Para o âmbito deste trabalho, o escopo da gerência definida pela NBR ISO/IEC 12207 foi limitado ao processo de desenvolvimento de software e aos processos de apoio. Dessa maneira, os processos organizacionais e os demais processos fundamentais foram considerados fora do escopo. Ademais, simplificaram-se as dependências do processo de desenvolvimento com os processos de apoio e organizacionais, mantendo-se o foco nas relações essenciais entre as atividades da gerência no processo de desenvolvimento.

A partir da NBR ISO/IEC 12207, pretende-se apresentar as recomendações seguindo as atividades prescritas pela norma: iniciação e definição do escopo, planejamento, execução e controle, revisão e avaliação e conclusão. A princípio, essas recomendações seriam referentes aos processos considerados adequados para um 
projeto, buscando seguir a forma como a norma define o processo gerencial. No entanto, tal abordagem apresentaria alguns pontos negativos do ponto de vista prático. De um lado, a gerência encarregada do processo de desenvolvimento seria responsável por um conjunto muito grande de atribuições. Do outro, alguns processos de apoio, que na prática são agrupados, seriam vistos como processos isolados. Com isso, considerouse mais interessante utilizar um conjunto de papéis baseado no Rational Unified Process (RUP) [30], usando-os como referência para uma divisão das atribuições de um gerente de projetos. Dessa forma, foram considerados os seguintes papéis gerenciais: gerente de projetos, gerente de controle de mudanças, gerente de configuração, gerente de testes, gerente de implantação, engenheiro de processos e revisor de gerência.

Além desses papéis gerenciais, existem também quatro outros papéis que são responsáveis pela gerência de escopo do projeto, materializada na gerência dos requisitos do sistema [7] [30]: analista de sistemas, arquiteto, especificador de requisitos e revisor técnico. Dessa forma, é proposto um outro papel gerencial que absorveria essas atribuições dispersas entre esses papéis: o gerente de requisitos.

Tabela 1: Os papéis gerenciais encarregados dos processos definidos pela NBR ISO/IEC 12207.

\begin{tabular}{|l|l|}
\hline Processo a ser gerenciado & \multicolumn{1}{|c|}{ Papel Relacionado } \\
\hline Desenvolvimento & $\begin{array}{l}\text { Gerente de projetos, gerente de testes, gerente de implantação, } \\
\text { engenheiro de processos e gerente de requisitos }\end{array}$ \\
\hline Documentação & Gerente de projetos, gerente de configuração e engenheiro de processos \\
\hline Gerência de Configuração & Gerente de controle de mudanças e gerente de configuração \\
\hline Garantia de Qualidade & Gerente de projetos \\
\hline Verificação & Todos os papéis \\
\hline Validação & Gerente de projetos, gerente de implantação e revisor de gerência \\
\hline Revisão Conjunta & Todos os papéis \\
\hline Auditoria & Todos os papéis \\
\hline Resolução de Problemas & Todos os papéis \\
\hline
\end{tabular}

Usando a NBR ISO/IEC 12207 como referência das atividades da gerência de projetos, definiu-se para cada um dos papéis a parte das responsabilidades gerenciais da qual o papel seria encarregado. Muitas vezes os papéis têm responsabilidades provenientes de mais de um processo. O engenheiro de processo, por exemplo, é responsável por parte da gerência do desenvolvimento e também por parte da gerência da documentação. Dessa maneira, um mapeamento dos papéis definidos a partir do RUP em relação à cobertura dos processos escolhidos da NBR ISO/IEC 12207 é apresentado na Tabela 1, fundamentado nas atividades definidas pelo padrão e pelas atividades propostas pelo RUP. Como os papéis servem apenas como referência para agrupar um conjunto de atividades, é importante notar que esse mapeamento extrapola o que é definido pelo RUP para que os papéis possam ser cobertos pela norma. Maiores detalhes deste mapeamento podem ser vistos em [32].

\subsection{O Método}

Para que sejam feitas recomendações é necessário que haja alguma forma de experiência envolvida. No caso do CMM, por exemplo, a criação de um conjunto de recomendações (ou melhor, práticas para a melhoria do processo) foi feita a partir da experiência obtida através do julgamento de diversos projetos e também das informações retro-alimentadas pelo governo e pela indústria [28]. Para as recomenda- 
ções aqui criadas, a principal fonte de experiência são os problemas e soluções extraídas de diversos relatos de experiência. A partir disso, as recomendações são criadas para cada uma das tarefas realizadas por cada um dos papéis gerenciais (considerando o mapeamento da NBR ISO/IEC 12207 no conjunto de papéis definidos a partir do RUP). Na Figura 1 é apresentada uma visão geral do método usando um diagrama BPMN [5].

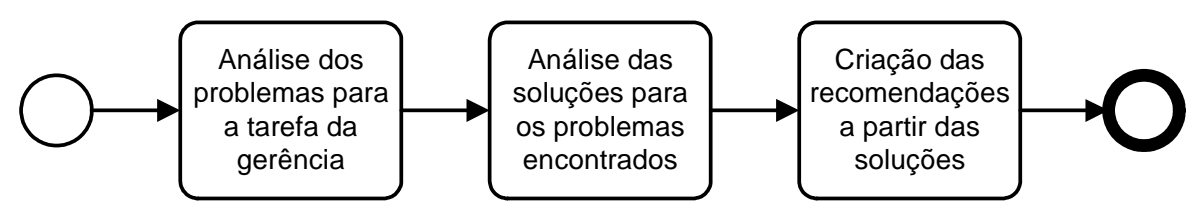

Figura 1: Visão geral do método para a criação das recomendações.

Para uma determinada tarefa, são analisados os problemas que poderiam influenciar a sua realização, usando como embasamento alguns problemas apontados pela literatura. É assim analisado como cada uma das características do DDS poderia causar dificuldades para essa determinada tarefa. A partir dessa lista de possíveis problemas buscou-se na literatura soluções e recomendações que tratassem especificamente desses problemas. Nos casos em que não havia nenhuma solução ou recomendação na literatura, tentou-se generalizar o problema (ou o conjunto deles) e observar o que o causava, criando assim uma recomendação a partir dessa análise. Por fim, algumas recomendações foram simplificadas e agrupadas, buscando criar um conjunto mínimo que tratasse de todos os problemas.

\section{As Recomendações}

Espera-se que as recomendações auxiliem o trabalho da gerência ao ressaltar algumas considerações que se tornam importantes devido à distribuição do desenvolvimento de software. Mas, antes que um gerente aplique essas recomendações, é necessário entender como é o projeto em relação ao DDS. Um projeto em que as pessoas estão distribuídas em dois grupos, um na Índia e o outro Brasil, será completamente diferente de um projeto em que cada parte envolvida está localizada em uma cidade do estado de São Paulo, por exemplo. Algumas das recomendações provavelmente não serão suficientemente interessantes para a gerência considerar em determinados casos. A cultura regional, por exemplo, pode ser pouco importante para o projeto em que as pessoas estão no mesmo estado. Da mesma forma, um gerente de testes pode considerar o agrupamento pouco importante, já que as pessoas responsáveis pelos testes e os desenvolvedores estão em um mesmo local, apesar de as outras partes envolvidas estarem em um outro país. Com isso, é necessário que cada papel gerencial analise a importância de cada uma das recomendações ao considerar as características do DDS [31] do projeto em questão. Cabe ao gerente analisar se deve ou não seguir uma determinada recomendação, seja ao ponderar o custo, o prazo, o escopo e a qualidade ou qualquer outra questão relativa ao projeto. O não atendimento a uma determinada recomendação, não quer dizer obrigatoriamente que o projeto sofrerá com o problema que a gerou. Da mesma forma, só porque uma recomendação foi seguida não quer dizer que o projeto estará livre desse problema. As recomendações são propostas de soluções criadas a partir da análise de problemas e soluções apontadas pela literatura.

Considerando essas observações, a seguir são apresentadas as recomendações para os papéis de gerente de requisitos e de engenheiro de processos, para os processos gerenciais relacionados ao processo de desenvolvimento. As recomendações estão 
organizadas por atividade, seguindo a NBR ISO/IEC 12207, e por tarefas, seguindo o mapeamento da norma com os papéis da gerência de projetos baseados no RUP (os números usados - ex: 5.3.1.3 - correspondem à identificação de tarefas definidas pela norma e em parênteses são apresentadas as tarefas gerenciais definidas pela norma que são tratadas pela tarefa proposta para o papel gerencial). Em alguns casos as recomendações foram baseadas em lições aprendidas (e até recomendações) apresentadas em diversos trabalhos - seja de forma direta ou para um caso específico que foi aqui generalizado.

\subsection{Recomendações para o Gerente de Requisitos}

\section{Iniciação}

a. Estabelecer os requisitos do desenvolvimento em relação à gerência dos requisitos conforme a tarefa 5.3.1.3: selecionar os padrões, ferramentas, etc, para a execução da gerência de requisitos (7.1.1.1).

1. Considerar a necessidade de participação no processo de pessoas de uma determinada cultura.

2. Considerar a necessidade de viagens durante a execução das atividades.

3. Considerar a necessidade de pessoas com domínio adequado da língua utilizada no local (ou definida).

4. Considerar a necessidade de documentos em uma determinada língua.

5. Considerar a necessidade de treinamento em uma determinada língua.

6. Considerar a necessidade de obtenção de um consenso sobre o processo e métodos empregados.

7. Considerar a necessidade de segurança das informações trafegadas (e como garantir).

8. Considerar as necessidades para a divulgação dos documentos.

9. Considerar a necessidade de infra-estrutura de comunicação para realização adequada das atividades.

10. Considerar as necessidades para divulgação de informações devido à relação legal existente.

b. Estabelecer a viabilidade do desenvolvimento em relação à gerência de requisitos (7.1.1.2).

1. Analisar se o tempo necessário (ao considerar esses recursos) é adequado devido à demora de decisões e discussões à distância - além de possíveis problemas de fuso horário.

2. Analisar se as necessidades são adequadas considerando as culturas regionais e organizacionais envolvidas.

3. Analisar a viabilidade de viagens de funcionários devido à necessidade de vistos.

4. Analisar as leis e custos de importação e exportação.

5. Analisar a possibilidade de uso das ferramentas idealizadas devido a infraestrutura do local.

6. Analisar as questões legais e de suporte global das ferramentas a serem usadas.

7. Analisar a existência de infra-estrutura adequada nos locais.

c. Modificar os requisitos da gerência de requisitos conforme necessário e possível (7.1.1.3). 
- Não são propostas recomendações.

Planejamento

a. Planejar a gerência dos requisitos conforme a tarefa 5.3.1.4 (7.1.2.1).

1. Considerar o agrupamento para a divisão do trabalho.

2. Considerar a necessidade de realização de reuniões em proximidade física.

3. Considerar o tempo de viagem das pessoas [3].

4. Garantir o uso de documentos iguais durante teleconferências.

5. Considerar a dificuldade de agendar reuniões.

6. Considerar as diferenças de horário de trabalho.

7. Fazer um rodízio dos locais que devem ser prejudicados no horário de realização de reuniões [3] [10].

8. Estudar a cultura local [6] [15].

9. Considerar a dificuldade de participação de reuniões devido à fluência em um idioma.

10. Considerar a necessidade de traduzir documentos.

11. Considerar as diferenças de calendário, feriados e ano legal [2].

12. Considerar o tempo de importação e exportação (alfândega) [3].

13. Definir claramente as formas de avaliação e trabalho das pessoas de diferentes organizações.

14. Padronizar (ou pelo menos mapear) os nomes de artefatos.

15. Padronizar os documentos usados [25].

16. Considerar o tempo para transferência de dados (ou até sobrecarga na rede, dependendo da quantidade de informações) [15].

17. Considerar o custo com investimento em infra-estrutura.

18. Mesmo que os processos empregados sejam diferentes, definir aspectos de alto nível que devam ser comuns [3] [6] [18].

19. Definir as ferramentas usadas, ou formatos que sejam compatíveis [15] [19].

20. Realizar teleconferências para discussões.

21. Definir em contrato as responsabilidades e a propriedade intelectual [19] [21].

\section{Execução e controle}

a. Executar os planos (7.1.3.1).

- Não são propostas recomendações.

b. Realização da gerência de requisitos através das atividades 5.3.2 e 5.3 .4 (7.1.3.1).

1. Confirmar os requisitos diretamente com as partes envolvidas, no caso do uso de intermediários.

2. Considerar a dificuldade de negociação dos requisitos devido à separação das pessoas.

3. Realizar a primeira reunião em proximidade física [15] [27].

4. Considerar a necessidade de pessoas fluentes na língua de uma determinada parte envolvida.

5. Considerar a existência de requisitos específicos a uma determinada cultura.

6. Considerar a possível diferença de interpretação de requisitos.

c. Realização da gerência de requisitos ao gerenciar as tarefas 5.3.3.2, 5.3.5.2, 5.3.6.7, 5.3.7.5 e 5.3.8.5 (7.1.3.1).

1. Fomentar a comunicação (formal e informal) entre as partes. 
2. Definir e divulgar qual é a pessoa encarregada [25].

3. Permitir sobreposição nos horários de trabalho.

d. Monitorar a execução do processo e informar as partes (7.1.3.2).

1. Desenvolver métricas de desempenho práticas [14].

2. Refinar as habilidades de estimativa (da equipe e do gerente) [14].

3. Aumentar a visibilidade com entregas freqüentes [14].

4. Oferecer a todos uma visão completa do que os demais estão fazendo [14].

e. Investigar, analisar e resolver os problemas descobertos durante a execução do processo, o que pode resultar em mudanças dos planos (devendo documentar tudo), conforme a tarefa 5.3.1.2 (7.1.3.3).

1. Uso de uma pessoa encarregada de relações públicas em cada local [3] [6] [21].

f. Relatar o andamento do desenvolvimento em relação aos requisitos, declarando aderência aos planos (7.1.3.4).

- Não são propostas recomendações.

$\underline{\text { Revisão e avaliação }}$

a. Garantir que os resultados e os produtos são avaliados para a satisfação dos requisitos (7.1.4.1).

1. Considerar as diferenças de percepção de não-conformidades devido a uma determinada cultura regional ou organizacional.

2. Definir padrões mínimos de qualidade [21].

b. Julgar os resultados da avaliação dos produtos, atividades e tarefas para a satisfação dos objetivos e completude dos planos (7.1.4.2).

- Não são propostas recomendações.

\section{Conclusão}

a. Determinar o fim da gerência dos requisitos (após as atividades, tarefas e produtos estarem completos) observando os critérios definidos em contrato ou procedimento da organização (7.1.5.1).

1. Definir em contrato o final da gerência de requisitos.

2. Advogados devem checar os contratos em relação às diferentes legislações [21].

b. Checar os resultados e registros por completude, posteriormente arquivando-os (7.1.5.2).

- Não são propostas recomendações.

\subsection{Recomendações para o Engenheiro de Processos}

$\underline{\text { Iniciação }}$

a. Definir o ciclo de vida e moldar processo segundo as tarefas 5.3.1.1 e 5.3.1.2 (7.1.1.1).

1. Realizar a integração com freqüência [3] [15].

2. Aumentar o formalismo do processo [22].

3. Considerar no processo as possibilidades do agrupamento existente.

4. Criar grupos multifuncionais e quase auto-suficientes (ciclo completo no local) [3] [9].

5. Divulgar o calendário das equipes [17] [27]. 
6. Divulgar o responsável e o objetivo da tarefa sendo realizada.

7. Divulgar a experiência dos funcionários [24] [27].

8. Fomentar a comunicação informal (ex: grupos de discussão e programas de mensagens instantâneas) [16].

9. Definir treinadores que atuem continuamente durante o projeto [9] [24].

10. Aproveitar a existência de sobreposição no horário de trabalho.

11. Observar se há alguma questão cultural que possa limitar a adoção do processo.

12. Mesmo que os processos empregados sejam diferentes, definir aspectos de alto nível que devam ser comuns [3] [6] [18].

13. Definir um vocabulário comum e produtos comuns (ou pelo menos um mapeamento entre eles) [3] [6] [15].

14. Considerar as limitações de passagem de conhecimento e comunicação devido à relação de negócio existente.

b. Selecionar os padrões, ferramentas, etc para a execução do processo segundo a tarefa 5.3.1.3 (7.1.1.1).

1. Definir protocolos para a comunicação [6] [14] [24] [27].

2. Considerar a necessidade de segurança das informações trafegadas (como garantir) [14] [19].

3. Analisar a necessidade de diversas formas de comunicação [21].

4. Definir um idioma padrão para o projeto.

5. Padronizar os documentos usados [25].

6. Observar se há alguma questão cultural que possa limitar a adoção de um padrão [15] [19].

7. Definir as ferramentas usadas, ou formatos compatíveis a todos [15] [19].

8. Analisar a possibilidade de uso das ferramentas idealizadas devido a infraestrutura do local.

9. Analisar as questões legais e de suporte das ferramentas a serem usadas [3].

Planejamento

a. Auxiliar o desenvolvimento de planos para conduzir as atividades do processo de desenvolvimento segundo a tarefa 5.3.1.4 (7.1.2.1).

- Não são propostas recomendações.

\section{Execução e controle}

a. O gerente deve investigar, analisar e resolver os problemas descobertos durante a execução do processo, o que pode resultar em mudanças dos planos (devendo documentar tudo), conforme a tarefa 5.3.1.2 (7.1.3.3).

1. Definir uma pessoa como encarregada de relações públicas em cada local [3] [6] [21].

\section{$\underline{\text { Revisão e avaliação }}$}

a. Julgar os resultados da avaliação dos produtos, atividades e tarefas para a satisfação dos objetivos (e completude dos planos) (7.1.4.2).

- Não são propostas recomendações.

\section{Conclusão}

a. Checar os resultados e registros quanto a completude, posteriormente arquivando-os (7.1.5.2). 
- Não são propostas recomendações.

\section{Estudo de Caso}

Para discutir e analisar o método proposto e as recomendações criadas, foi realizado um estudo de caso. O projeto estudado foi o desenvolvimento de um software de redução de dados para um espectrógrafo de alta resolução (instrumento óptico responsável pela separação da radiação obtida por um telescópio), organizado pelo Laboratório Nacional de Astrofísica (LNA). O LNA é uma das unidades de pesquisa do Ministério de Ciência e Tecnologia e tem como missão principal fornecer meios e infra-estrutura para que a comunidade astronômica brasileira possa realizar a sua pesquisa [23].

O projeto foi realizado por uma equipe distribuída entre três Estados brasileiros trabalhando em horários variados, dependendo da disponibilidade das pessoas. Apesar de não haver diferenças significativas de idioma, de cultura regional e de outros aspectos locais, as infra-estruturas variavam entre os locais, assim como as culturas organizacionais (forma de trabalho e processos empregados, principalmente). A relação de negócio variava entre as pessoas, havendo alguns que trabalhavam de forma voluntária e outros que recebiam bolsas para participar do projeto.

Para analisar o projeto foi formulado um questionário com 140 questões que pretendiam avaliar o que foi feito e como o DDS afetou essa realização, ressaltando os problemas ocorridos durante a execução das atividades de desenvolvimento da gerência de requisitos e da engenharia de processos. Para que essas informações fossem consistentes com o método proposto para a criação das recomendações, as questões seguiram o mapeamento da NBR ISO/IEC 12207 no conjunto de papéis baseados no RUP. Além disso, o questionário também tratou diretamente das recomendações à gerência de projetos ao questionar como as tarefas deveriam ter sido realizadas e o que deveria ser alterado. Como exemplo das questões formuladas, na Figura 2 são apresentadas as questões para a atividade de iniciação da gerência de requisitos.

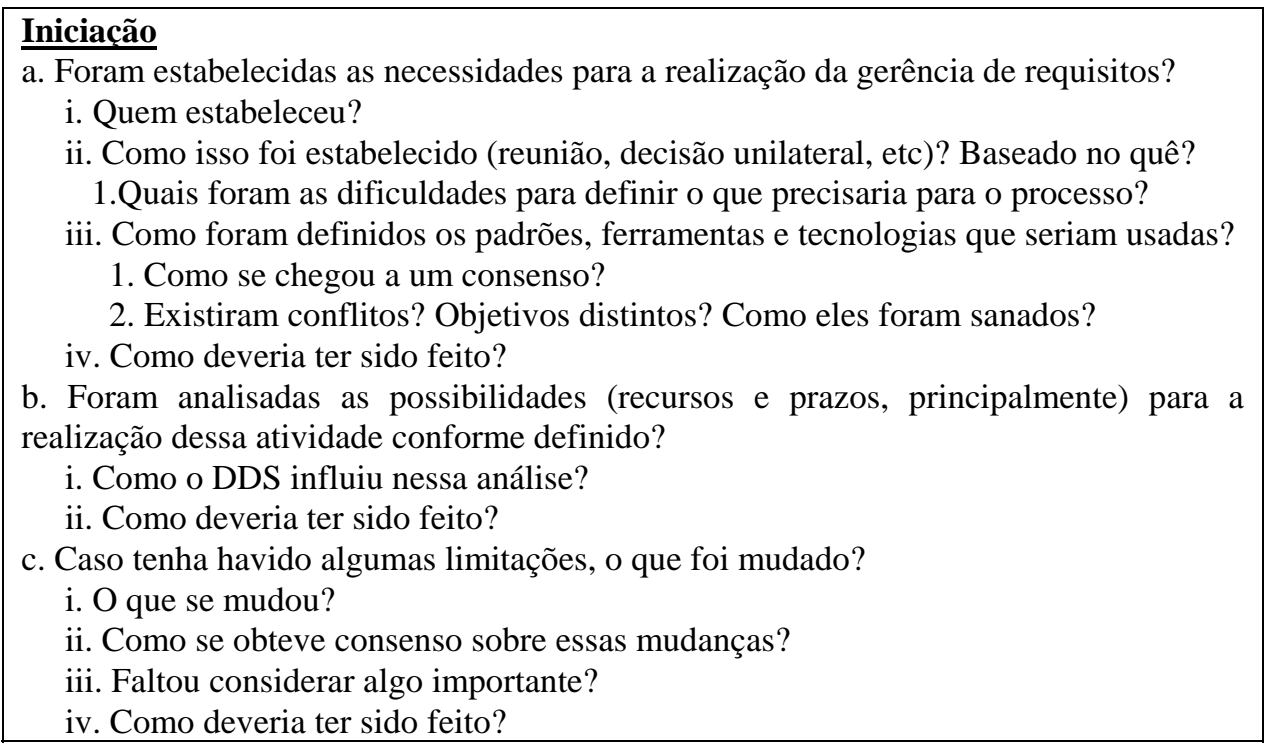

Figura 2: As questões formuladas para a análise da atividade de iniciação da gerência de requisitos. 
A análise das respostas permitiu observar que as atividades da gerência de requisitos e da engenharia de processos foram realizadas de modo informal, sempre buscando obter um consenso de todas as partes envolvidas (o que poderia demorar devido à distribuição das pessoas). Um mapeamento entre os problemas encontrados e as recomendações aqui propostas (referenciadas pela atividade, tarefa e número da recomendação) é apresentado na Tabela 2.

Tabela 2: Os problemas encontrados e as recomendações associadas (referenciadas pela atividade, tarefa e número da recomendação).

\begin{tabular}{|c|c|c|c|}
\hline Atividade & Papel & Problema & Recomendação \\
\hline \multirow{4}{*}{ Iniciação } & $\begin{array}{l}\text { Gerente de } \\
\text { Requisitos }\end{array}$ & $\begin{array}{l}\text { Orçamento não considerava recursos para } \\
\text { viagens }\end{array}$ & Nenhuma recomendação \\
\hline & $\begin{array}{l}\text { Engenheiro } \\
\text { de Processos }\end{array}$ & $\begin{array}{l}\text { Falta de consenso nos padrões de código e } \\
\text { nos softwares usados }\end{array}$ & - Iniciação, b-7 \\
\hline & $\begin{array}{l}\text { Engenheiro } \\
\text { de Processos }\end{array}$ & Infra-estrutura de comunicação inadequada & - Iniciação b-3 \\
\hline & $\begin{array}{l}\text { Engenheiro } \\
\text { de Processos }\end{array}$ & $\begin{array}{l}\text { Dependência de reuniões em proximidade } \\
\text { física }\end{array}$ & - Iniciação b-3 \\
\hline Planejamento & $\begin{array}{l}\text { Gerente de } \\
\text { Requisitos }\end{array}$ & Demora em obter consenso & Nenhuma recomendação \\
\hline \multirow{2}{*}{$\begin{array}{l}\text { Execução e } \\
\text { controle }\end{array}$} & $\begin{array}{l}\text { Gerente de } \\
\text { Requisitos }\end{array}$ & $\begin{array}{l}\text { Dificuldade de participação de algumas } \\
\text { pessoas nas reuniões }\end{array}$ & $\begin{array}{l}\text { - Planejamento a-5 } \\
\text { - Iniciação a-9 }\end{array}$ \\
\hline & $\begin{array}{l}\text { Gerente de } \\
\text { Requisitos }\end{array}$ & Dificuldade de discutir à distância & - Planejamento a-20 \\
\hline $\begin{array}{l}\text { Revisão e } \\
\text { avaliação }\end{array}$ & $\begin{array}{l}\text { Gerente de } \\
\text { Requisitos }\end{array}$ & Demora no uso do e-mail & - Iniciação a-9 \\
\hline Conclusão & & Nenhum problema apontado & \\
\hline
\end{tabular}

Ao analisar esse mapeamento, percebe-se que a maioria dos problemas está relacionada a pelo menos uma recomendação. Entretanto, houve dois problemas que não foram tratados pelas recomendações: o orçamento não considerava recursos para viagens e a demora em obter consenso. O primeiro problema sugere a criação de uma recomendação para a tarefa "estabelecer a viabilidade do desenvolvimento em relação à gerência de requisitos”: analisar os recursos para viagens. Para o segundo problema, a única sugestão que parece adequada é "planejar os conflitos inevitáveis” (proposto em [6]), já que não é possível evitar a demora. Dessa maneira, poderia ser criada essa recomendação para a atividade de planejamento. Além disso, para o problema "falta de consenso nos padrões de código e nos softwares usados” a recomendação relacionada só cobre parcialmente a questão dos softwares. Isso sugere a criação de uma recomendação para a tarefa de iniciação "selecionar os padrões, ferramentas, etc para a execução do processo": obter consenso sobre padrões de código.

Considerando as três recomendações sugeridas a partir dos problemas encontrados durante o estudo do projeto, percebem-se algumas dificuldades no método empregado. A não consideração da recomendação "obter consenso sobre padrões de código" mostra a dificuldade de absorver os problemas existentes e generalizá-los para a criação de recomendações, por mais que o problema tenha sido apontado por outros relatos de experiência. Isso demonstra que o método é dependente da interpretação e percepção de quem o aplica. De maneira semelhante, a não criação da recomendação "planejar por conflitos inevitáveis" aponta a dependência do método com a experiência (conhecimento de soluções adequadas) de quem o aplica. 


\section{Conclusão}

O DDS é um assunto bastante vasto e ainda pouco pesquisado, considerando a sua importância e complexidade. A tendência é que as organizações busquem mais freqüentemente a realização dessa forma de desenvolvimento, almejando aproveitar as diversas motivações e vantagens disponíveis. Conforme houver progresso nas tecnologias de comunicação, nas ferramentas de suporte e nas soluções práticas e teóricas em geral, os problemas existentes poderão ser mais bem gerenciados, tornando essa forma de desenvolvimento ainda mais atraente e importante.

Com o objetivo de auxiliar a realização das responsabilidades gerenciais em um desenvolvimento distribuído de software, neste artigo foram propostas recomendações ao gerente de requisitos e ao engenheiro de processos e, mais que isso, foi proposto um método para a criação dessas recomendações embasado no processo de gerência apresentado na NBR ISO/IEC 12207 e em um conjunto de papéis baseado no RUP.

Ao comparar os problemas observados empiricamente com as recomendações propostas foi possível observar que a maioria dos problemas observados no projeto estudado tinha uma recomendação associada. Entretanto, isso não garante que se as recomendações fossem aplicadas ao projeto esses problemas não aconteceriam, uma vez que outras recomendações talvez também sejam necessárias. A análise realizada também evidenciou que as recomendações precisam ainda acumular uma maior experiência. O método proposto e aplicado para a criação das recomendações depende da experiência, interpretação e percepção de quem o aplica. Para aplicar o método é necessário identificar adequadamente os principais problemas para uma determinada tarefa, generalizar esses problemas e absorver o conhecimento empírico e teórico para criar as recomendações. Até mesmo o questionário empregado exigia uma experiência suficiente para distinguir os problemas técnicos normais de projetos de desenvolvimento de software dos problemas causados pelo DDS.

Por fim, talvez a principal limitação da análise realizada foi a discussão de apenas um projeto, o que impossibilita uma validação ou uma conclusão mais definitiva, servindo apenas como uma investigação do assunto. De qualquer forma, espera-se que as recomendações e o método para criá-las auxiliem a realização das atividades gerenciais em futuros projetos.

\section{Agradecimentos}

Essa pesquisa foi financiada por uma bolsa de mestrado fornecida pela Escola Politécnica da Universidade de São Paulo e pelo CNPq. Agradecemos o total apoio dado pelo Dr. Bruno Vaz Castilho do LNA durante a realização do estudo de caso.

\section{Referências}

[1] ABNT - Associação Brasileira de Normas Técnicas. (1998). NBR ISO/IEC 12207 tecnologia de informação: processos de ciclo de vida de software. ABNT.

[2] Bass, M. e Paulish, D. (2004). "Global Software Development Process Research at Siemens”. In: International Workshop on Global Software Development, 3., Edinburgh. 
[3] Battin, R. D., Crocker, R., Kreidler, J. e Subramanian, K. (2001). "Leveraging Resources in Global Software Development”. IEEE Software, v.18, n.2, p.70-77, March/April.

[4] Boland, D. e Fitzgerald, B. (2004). “Transitioning from a Co-located to a Globally Distributed Software Development Team: A Case Study at Analog Devices Inc.”. In: International Workshop on Global Software Development, 3., Edinburgh.

[5] BPMI - Business Process Management Initiative. (2004). "Business Process Modeling Notation (BPMN)”, http://www.bpmn.org. Version 1.0.

[6] Carmel, E. (1999). Global Software Teams: Collaborating Across Borders and Time Zones. Prentice Hall.

[7] Charbonneau, S. (2004). "Software Project Management - A Mapping Between RUP and the PMBOK”. http://www.therationaledge.com, The Rational Edge, May.

[8] Damian, D. e Zowghi, D. (2003). “An insight into the interplay between culture, conflict and distance in globally distributed requirements negotiations”. In: Hawaii International Conference on System Sciences, 36., Hawaii.

[9] Ebert, C. e De Neve, P. (2001). “Surviving Global Software Development”. IEEE Software, v.18, n.2, p.62-69, March/April.

[10] Evaristo, J. R. e Scudder, R. (2000). “Geographically Distributed Project Teams: A Dimensional Analysis”. In: Hawaii International Conference on System Sciences, 33., Hawaii.

[11] Gaeta, M. e Ritrovato, P. (2002). "Generalised Environment for Process Management in Cooperative Software Engineering”. In: Annual International Computer Software and Applications Conference, 26., Oxford.

[12] Harrington, S. J. e Ruppel, C. P. (1999). "Telecommuting: A Test of Trust, Competing Values, and Relative Advantage”. IEEE Transactions on Professional Communication, v.42, n.4, p.223-239, December.

[13] Hawryszkiewycz, I. T. e Gorton, I. (1996). “Distributing the Software Process”. In: Australian Software Engineering Conference, Melbourne.

[14] Haywood, M. (1998). Managing virtual teams: practical techniques for high technology project managers. Artech House Publishers.

[15] Haywood, M. (2000). "Working in Virtual Teams: A Tale of Two Projects and Many Cities”. IT Professional, v.2, n.2, p.58-60, March/April.

[16] Herbsleb, J. D. e Mockus, A. (2003). "An Empirical Study of Speed and Communication in Globally Distributed Software Development”. IEEE Transactions on software engineering, v.29, n.6, p.481-494, June.

[17] Herbsleb, J. D., Mockus, A., Finholt, T. A. e Grinter, R. E. (2000). "Distance, Dependencies, and Delay in a Global Collaboration”. In: ACM Conference on Computer Supported Cooperative Work, Philadelphia.

[18] Herbsleb, J. e Moitra, D. (2001). “Global Software Development”. IEEE Software, v.18, n.2, p.16-20, March/April. 
[19] Karolak, D. W. (1998). Global Software Development: Managing Virtual Teams and Environment. IEEE Computer Society Press.

[20] Kiel, L. (2003). “Experiences in Distributed Development: A Case Study”. In: International Workshop on Global Software Development, Portland.

[21] Kobitzsch, W., Rombach, D. e Feldmann, R. L. (2001) “Outsourcing in India”. IEEE Software, v.18, n.2, p.78-86, March/April.

[22] Kroll, P. e Kruchten, P. (2003). The Rational Unified Process Made Easy: a Practitioner's Guide to the RUP. Addison-Wesley.

[23] Laboratório Nacional de Astrofísica (2004). "Sobre o LNA", http://www.lna.br/lna/lna_hist.html.

[24] Layzell, P., Brereton, O. P. e French, A. (2000). "Supporting Collaboration in Distributed Software Engineering Teams”. In: Asia-Pacific Software Engineering Conference, 7., Singapore.

[25] Lopes, L. T. e Audy, J. L. N. (2003). “Em busca de um modelo de referência para engenharia de requisitos em ambientes de desenvolvimento distribuído de software”. In: Workshop Em Engenharia de Requisitos, 6., Piracicaba.

[26] Maidantchik, C. e Da Rocha, A. R. C. (2002). "Managing a Worldwide Software Process”. In: International Workshop on Global Software Development, Orlando.

[27] Mockus, A. e Herbsleb, J. D. (2001). "Challenges of Global Software Development”. In: International Software Metrics Symposium, 7., London.

[28] Paulk, M., Curtis, B., Chrissis, M. B. e Weber, C. V. (1993). Capability Maturity Model for Software. Technical Report CMU/SEI-93-TR-024. Carnegie Mellon Software Engineering Institute.

[29] Project Management Institute. (2000). A guide to the project management body of knowledge (PMBOK guide). Project Management Institute.

[30] Rational Unified Process. (2003). Version 2003.06.12.01. Rational Software Corporation.

[31] Siqueira, F. L. e Muniz Silva, P. S. (2004). “As Características do Desenvolvimento Distribuído de Software”. In: Simpósio Brasileiro de Sistemas de Informação, Porto Alegre,.

[32] Siqueira, F. L. e Muniz Silva, P. S. (2005). “Mapeamento da NBR ISO 12207 em Papéis da Gerência de Projetos de Software”. In: Simpósio Brasileiro de Qualidade de Software, Porto Alegre.

[33] Zanoni, R. e Audy, J. L. N. (2003). “Project Management Model for a Physically Distributed Software Development Environment”. In: Hawaii International Conference on System Sciences, 36., Hawaii. 